\title{
BMJ Open Efficacy of neoadjuvant atezolizumab treatment in patients with advanced urothelial bladder cancer according to the BASQ classification: a study protocol for an open-label, two-cohort, phase II trial
}

Hyeong Dong Yuk, ${ }^{1,2}$ Chang Wook Jeong, ${ }^{1,2}$ Cheol Kwak, ${ }^{1,2}$ Hyeon Kim, ${ }^{1,2}$ Kyung Chul Moon, ${ }^{2,3}$ Ja Hyeon Ku (i) 1,2

To cite: Yuk HD, Jeong CW, Kwak C, et al. Efficacy of neoadjuvant atezolizumab treatment in patients with advanced urothelial bladder cancer according to the BASQ classification: a study protocol for an open-label, two-cohort, phase II trial. BMJ Open 2020;10:e035530. doi:10.1136/ bmjopen-2019-035530

- Prepublication history and supplemental material for this paper are available online. To view these files, please visit the journal online (http://dx.doi. org/10.1136/bmjopen-2019035530).

Received 05 November 2019 Revised 18 August 2020 Accepted 21 September 2020

Check for updates

(c) Author(s) (or their employer(s)) 2020. Re-use permitted under CC BY-NC. No commercial re-use. See rights and permissions. Published by BMJ.

${ }^{1}$ Urology, Seoul National University Hospital, Seoul, The Republic of Korea

${ }^{2}$ College of Medicine, Seoul National University, Seoul, The Republic of Korea

${ }^{3}$ Pathology, Seoul National University Hospital, Seoul, The Republic of Korea

Correspondence to Dr Ja Hyeon Ku; kuuro70@snu.ac.kr

\section{ABSTRACT}

Introduction Atezolizumab is a programmed death ligand-1 inhibitor for urothelial bladder cancer treatment. Atezolizumab has become the standard therapy for patients with urothelial bladder cancer who are not responding to cisplatin-based chemotherapy and is also used as a first-line treatment in cisplatin-ineligible patients. However, the efficacy of atezolizumab as a neoadjuvant chemotherapy for radical cystectomy has not yet been published and is still under study. This trial investigates the effectiveness of basal/squamouslike (BASQ) classification in the selection of an effective target group of patients with muscle-invasive bladder cancer (MIBC) for neoadjuvant atezolizumab treatment.

Methods and analysis This study is an open-label, twocohort, phase II trial. It was designed to evaluate the efficacy of neoadjuvant atezolizumab treatment in patients with MIBC (T2-4NOM0) pathological responses after neoadjuvant chemotherapy and radical cystectomy. According to the molecular subtype characteristics of previous transurethral resection of the bladder specimens, patients are divided into two groups: luminal type (KRT5/6-KRT14-F0XA1+GATA3+) and basal type (KRT5/6+KRT14+F0XA1-GATA3-). Every 3 weeks, atezolizumab is administered at a dose of $1200 \mathrm{mg}$ for three cycles prior to radical cystectomy in patients with MIBC. The primary end point is objective pathological responses in the intention-to-treat patients. The secondary end point is a 1-year progression-free survival difference according to the BASQ classification in patients who underwent neoadjuvant atezolizumab treatment.

Ethics and dissemination The study protocol was approved by the Institutional Review Board of Seoul National University Hospital, Seoul, Republic of Korea (H 1806-051-950). The trial is registered at ClinicalTrials.gov. The trial results will be published in peer-reviewed journals and at conferences. Trial registration number NCT03577132.

\section{INTRODUCTION}

Blocking programmed cell death protein-1 (PD-1) and programmed death ligand-1 (PDL1) is an effective way to treat advanced-stage
Strengths and limitations of this study

- This is the first open-label, two-cohort, phase II trial to explore the effect of neoadjuvant immune checkpoint inhibitors on molecular subtypes in urothelial bladder cancer.

- The trial is designed to evaluate the efficacy of neoadjuvant atezolizumab treatment in patients with muscle-invasive bladder cancer (T2-4NOM0).

- It is also designed to evaluate the pathological responses to neoadjuvant atezolizumab treatment and radical cystectomy according to the basal/ squamous-like classification.

- The purpose of this study is to select a target group for which neoadjuvant immune checkpoint inhibitors may be more effective.

- The limitation of this study is the shorter follow-up period.

urothelial bladder cancer (UBC). ${ }^{1-3}$ Atezolizumab was first approved by the US Food and Drug Administration in May 2016 as a PD-L1 inhibitor for UBC. ${ }^{4}$ This new drug has become the standard therapy for patients with UBC who are not responding to cisplatinbased chemotherapy and is also used as a first-line treatment in cisplatin-ineligible patients. ${ }^{5}$ Several treatments are currently being studied, and in addition to cisplatinbased chemotherapy as the first-line neoadjuvant treatment for advanced UBC, it is reported that the benefits of immune checkpoint inhibitors are positive. ${ }^{6}$ In the PURE-01 (Pembrolizumab as Neoadjuvant Therapy Before Radical Cystectomy in Patients With Muscle-Invasive Urothelial Bladder Carcinoma) study to determine the activity of neoadjuvant pembrolizumab in 50 patients 
with T2-T3bN0 UBC, 21 patients showed a pathological complete remission (pCR) rate of $42 \%$ (95\% CI: $28.2 \%$ to $56.8 \%) .{ }^{7}$ In the ABACUS ${ }^{8}$ study of neoadjuvant atezolizumab in patients with T2-T4aN0M0 cisplatin chemotherapy inability, the pCR rate was $29 \%$ (95\% CI: $19 \%$ to $42 \%){ }^{89}$

Recently, several research groups have conducted a detailed analysis of the molecular genetic characteristics of bladder cancer through The Cancer Genome Atlas (TCGA) study and are working to apply it to UBC treatment. ${ }^{10-13}$ A consensus was reached regarding the existence of a group of basal squamous-like tumours designated basal/squamous-like (BASQ) characterised by the high expression of KRT5/6 and KRT14 genes and the low/undetectable expression of FOXA1 and GATA3 genes. ${ }^{14}$ This novel molecular classification can improve the identification of optimal patient populations for different treatment modalities. Specifically, luminal type and basal type may have different treatment responses and prognosis after initial definitive treatment, such as neoadjuvant treatment. ${ }^{14}$ However, there is not much evidence for this topic, particularly the clinical efficacy of neoadjuvant PD-L1 inhibitors according to the BASQ classification in patients with advanced UBC. This study assesses the efficacy of the neoadjuvant atezolizumab treatment in patients with muscle-invasive bladder cancer (MIBC) and the difference in the efficacy of neoadjuvant atezolizumab treatment according to the BASQ classification.

\section{METHODS AND ANALYSIS \\ Research design}

This study is an open-label, two-cohort, phase II trial for patients with histologically confirmed muscle-invasive UBC. It was designed to evaluate the efficacy of neoadjuvant atezolizumab treatment in patients with locally advanced or metastatic UBC pathological responses (pT0 change) after neoadjuvant chemotherapy and radical cystectomy.

\section{Study hypothesis and objectives}

The main hypothesis of this study is that patients with locally advanced or metastatic UBC can tolerate a neoadjuvant atezolizumab dosing regimen and that there will be differences in the effect of neoadjuvant immunotherapy between basal and luminal subtypes on immunohistochemistry (IHC), according to their BASQ classification.

The main objective of this study is to investigate and compare the efficacy and clinical responses (pT0 change) to neoadjuvant atezolizumab treatment in patients with MIBC according to their BASQ classification.

In patients with MIBC, the basal type is expected to have better efficacy and clinical responses to neoadjuvant atezolizumab treatment than the luminal type.

\section{Study end point}

The primary end point is objective pathological responses (pT0 change) in the intention-to-treat patients. After
Box 1 Key end points and inclusion criteria of study

\section{Primary end point}

- Objective pathological responses (pT0 change) after neoadjuvant atezolizumab treatment.

Secondary end point

- One-year progression-free survival difference according to the basal/squamous-like (BASQ) classification of patients who underwent neoadjuvant atezolizumab treatment.

\section{Inclusion criteria}

$>\geq 18$ years of age.

- Histologically confirmed muscle-invasive urothelial carcinoma.

- Patients undergoing radical cystectomy.

- Advanced status requiring neoadjuvant systemic therapy.

- Eastern Cooperative Oncology Group performance status score of 0 or 1.

- Adequate organ and haematological functions.

Available immunohistochemistry data for the BASQ classification.

neoadjuvant treatment with atezolizumab in patients with advanced MIBC, objective pathological responses are compared according to the BASQ classification. The second end point is 1-year progression-free survival (PFS) difference according to the BASQ classification of patients who underwent neoadjuvant atezolizumab treatment (box 1). PFS is defined as the time between the date of first documented disease progression or death, whichever occurs first. Disease progression is determined on the basis of investigator assessment with the use of Response Evaluation Criteria In Solid Tumours version 1.1 (RECIST v1.1). Patients who would not have experienced disease progression or death at the time of analysis will be censored at 1 year after treatment.

Safety assessments are performed for 90 days after the last atezolizumab administration in accordance with National Cancer Institute Common Terminology Criteria for Adverse Events (NCI CTCAE), version 4.03.

\section{Safety item and measurement}

Adverse reactions due to the injection of anticancer drugs are as follows: adverse events reported in clinical trials, immune-mediated adverse events, infection, infusionrelated reactions and immunogenicity. The safety assessment is performed from the beginning of the study up to 90 days after the last injection of atezolizumab, in accordance with NCI CTCAE, version 4.03. The incidence, characteristics and severity of side effects are graded in accordance with NCI CTCAE, version 4.0, and changes in biometric signals, physical examination results and clinical examination results are identified.

Safety monitoring was conducted at the time of registration of five research subjects. All subjects were followed up on a regular basis. When an adverse reaction occurred, the adverse reaction content, seriousness, predictability and causal relationship were evaluated and reported. In the case of serious adverse events, they were immediately reported to the Subject Protection Centre. 


\section{Study population}

A total of 40 patients with MIBC undergoing radical cystectomy following neoadjuvant treatment with atezolizumab are to be included. All patients must have undergone transurethral resection of the bladder (TURB) before radical cystectomy. According to the molecular subtype characteristics of previous TURB specimens, we divided the patients according to the BASQ classification (KRT5/6, KRT14, FOXA1 and GATA3) into two groups: luminal type (KRT5/6-KRT14-FOXA1+GATA3+) and basal type (KRT5/6+KRT14+FOXA1-GATA3-). A total of 40 patients, 20 for each group, were included in the study.

\section{BASQ classification}

The BASQ classification is a consensus conclusion agreed by multicentre researchers who conducted TCGA studies. ${ }^{10-1315}$ According to these classification criteria, invasive bladder cancer subtypes are divided into basal type and luminal type. The basal type is characterised by extensive expression of KRT5/6 and KRT14 and low expression levels of FOXA1 and GATA3 at the RNA and protein levels. KRT5/6 and KRT14 are extensively expressed in cancer cells without epithelial compartmentalisation. ${ }^{10-13} 15$ The basal type is associated with a high resistance to chemotherapy and poor prognosis. ${ }^{14}$

\section{Inclusion criteria}

Inclusion criteria for the study are the following: $(1) \geq 18$ years of age; (2) histologically confirmed muscle-invasive urothelial carcinoma; (3) patients undergoing radical cystectomy; (4) advanced status requiring neoadjuvant systemic therapy; (5) Eastern Cooperative Oncology Group performance status score of 0 or 1; (6) adequate organ and haematological functions; and (6) available IHC data for the BASQ classification (box 1).

\section{Exclusion criteria}

Exclusion criteria for the study are the following: (1) non-urothelial carcinoma histology; (2) active autoimmune disease or inflammatory bowel disease; (3) prior severe or persistent immune-related adverse events; (4) previous exposure to anti-PD-1 or anti-PD-L1 therapy; (5) requirement for $10 \mathrm{mg} /$ day of prednisone or equivalent; (6) inadequate liver, kidney function, and hematological dysfunction; (7) inoperable case, such as untreated central nervous system metastases; and (8) no available archival tumour tissue for evaluating the BASQ classification.

\section{Sample size consideration}

The efficacy of atezolizumab as a neoadjuvant chemotherapy for radical cystectomy has not yet been published and is still under study. This study is a pilot study concept. To maximise its excellence in research progress and feasibility, we set the number of patients prospectively to 40 , divided into two groups of 20 . To detect a difference with $80 \%$ power and $5 \%$ significance level, we needed at least about 15 patients per cohort. About $80-100$ patients had undergone radical cystectomy in our institution. We considered the number of patients diagnosed with MIBC

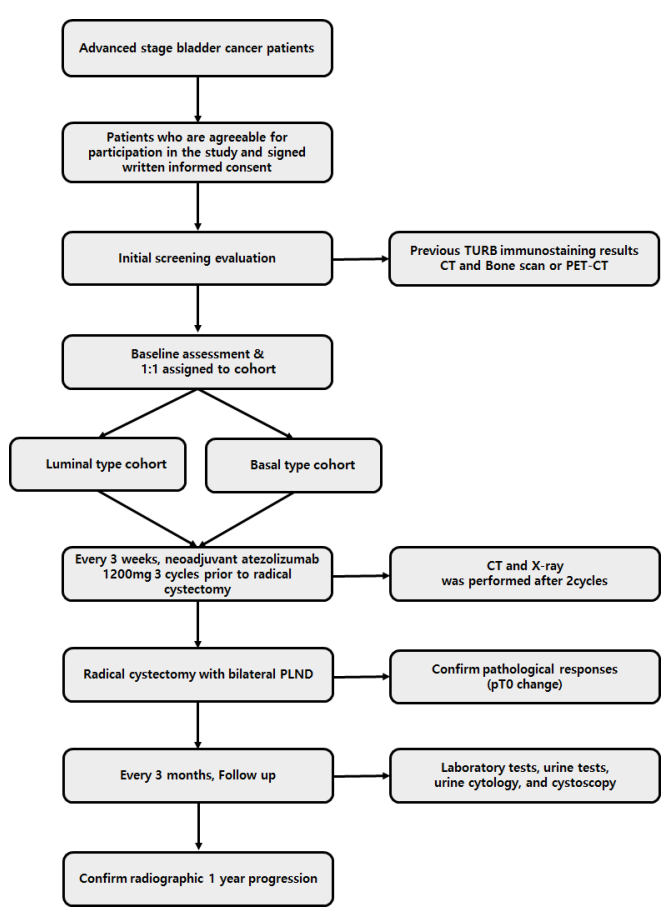

Figure 1 Study flow diagram. PET, positron emission tomography; PLND, pelvic lymphadenectomy; TURB, transurethral resection of the bladder.

who had undergone TURB in our institution and who could have a BASQ classification. Considering patients' refusal to take part in the study and drop out of the study, about 40 patients are considered to be able to participate in the study for about $3-4$ years.

\section{Drug regimens}

The dose level of atezolizumab in this study is $1200 \mathrm{mg}$ (equivalent to an average body-weight-based dose of $15 \mathrm{mg} / \mathrm{kg}$ ) administered by intravenous infusion every 3 weeks (21 days). The initial dose of atezolizumab is delivered over $60 \mathrm{~min}$. If the first infusion is tolerated without infusion-associated adverse events, the second and third infusion will be delivered over 30 min every 3 weeks, for a total of three cycles prior to radical cystectomy.

\section{Data collection}

The studies were planned following the flow shown in figure 1. Screening tests included CT of the chest, abdomen, and pelvis. Bone scans or positron emission tomography/CT was performed at the time of screening, when necessary. Previous TURB specimens of all patients underwent immunostaining with KRT5/6, KRT14, FOXA1 and GATA3 antibodies using tissue microarray (TMA), and the BASQ classification results were obtained according to the immunostaining expression pattern.

At the time of screening, we collected information on clinical tumour, node, metastases (TNM) stage, tumour grade, BASQ classification information, American Society of Anesthesiologist physical status, previous medical history, haematology, serum chemistries, coagulation and serology. 
After two cycles of neoadjuvant atezolizumab, CT scan and X-ray analyses were performed. After radical cystectomy, pathological TNM stage, tumour grade, positive surgical margin and lymphovascular invasion were determined. The follow-up was performed every 3 months after radical cystectomy, and progression was confirmed by CT scan 1 year later. Laboratory tests, urine tests, urine cytology and bladder cystoscopy were performed at each follow-up. Radiological tests such as chest, abdomen and pelvis CT were performed 1 year after operation. We also collected various types of oncological data, including recurrence, progression, mortality and cancer-related mortality rates.

\section{TMA construction and IHC}

We constructed TMA blocks from formalin-fixed paraffinembedded tissue blocks (Superbiochips Laboratories, Seoul, Korea). In brief, two representative tumour cores $(2 \mathrm{~mm}$ in diameter) were selected from the viable tumour area. The cancer tissues of patients were examined microscopically by a skilled pathologist, and the TMA was prepared after selecting the most representative cancer tissues. Immunostaining was performed for KRT5/6, KRT14, FOXA1 and GATA3 antibodies on TMA slides, and the expression patterns were quantitatively analysed using a scanning programme. Based on the expression patterns, the patients were divided according to the BASQ classification (KRT5/6, KRT14, FOXA1 and GATA3).

IHC staining was performed on $4 \mu \mathrm{m}$ thick sections of TMA blocks using the Benchmark XT autostainer (Ventana Medical Systems, Tucson, Arizona, USA). The sections were incubated with the following primary antibodies: mouse monoclonal antibodies against KRT5/6 (64 min; 1:50; Dako, Glostrup, Denmark), KRT14 (32min; 1:50; Cell Marque, Rocklin, California, USA) and GATA3 (32min; 1:500; clone 156-3C11; Cell Marque), and rabbit polyclonal antibody against FOXA1 (16min; 1:700; ThermoFisher Scientific, Rockford, Illinois, USA).

\section{Statistical analysis}

Objective pathological responses were assessed by wellexperienced genitourinary pathologists. Comparisons between BASQ groups were made via t-tests and Fisher's exact tests. Continuous variables are presented as median value and IQRs or average value and SDs. Nominal variables are presented as the frequency of events (\%). The Kaplan-Meier method was used to predict PFS, and significance among groups was determined using the log-rank tests. Safety analyses were performed on the as-treated population, defined as all enrolled patients who received atezolizumab. We used the logistic model to analyse the association between BASQ and pT0 response.

All statistical tests were performed using IBM SPSS Statistics V.25.0 (IBM) and STATA V.14 (StataCorp LP, College Station, Texas, USA). A p value $<0.05$ was considered statistically significant.

\section{Patient and public involvement}

Patients and/or the public were not involved in the design, recruitment and conduction of this study.

\section{DISCUSSION}

Unlike studies of other neoadjuvant settings of immune checkpoint inhibitors, this study combines a neoadjuvant immunotherapy with a molecular subtype, which is clinically and theoretically innovative. First, unlike the ABACUS and PURE-01 studies, the purpose of our study was to select a target group for which neoadjuvant chemotherapy may be more effective. Second, this is a two-cohort study with prospective molecular subtypes. The ABACUS and PURE-01 studies are single-arm studies. Third, in the ABACUS study, cancers are retrospectively classified as TCGA subtypes to suggest an effective molecular subtype. Finally, there is a difference between the drug and the regimen used. Pembrolizumab is used in the PURE-01 study, and two cycles of neoadjuvant atezolizumab are used in the ABACUS study. According to our knowledge, this study is the first open-label, parallelgroup, controlled clinical trial to explore the effect of neoadjuvant immune checkpoint inhibitors on molecular subtypes in bladder cancer. This will contribute to the development of an effective neoadjuvant immunotherapy for patients with MIBC. This study will provide us unique opportunities to identify patients and to predict better outcomes before neoadjuvant immunotherapy.

In addition, this study also provides an opportunity to further study neoadjuvant immunotherapy and personalised care in patients with MIBC. It can be an opportunity to provide better explanatory data on the neoadjuvant immunotherapy theory. The effects of immunotherapy on individual molecular characteristics can be used as a basis for changing the current consensus guideline recommendations.

Atezolizumab is a humanised monoclonal anti-PD-L1 antibody and has demonstrated efficacy and safety as a first-line treatment in cisplatin-ineligible patients with locally advanced and metastatic bladder cancer in the phase II IMvigor210 trial. ${ }^{16}$ The primary outcome was an objective response rate of $23.5 \%$ (95\% CI: $16.2 \%$ to $32.2 \%$ ) in the group receiving atezolizumab. ${ }^{16}$ Researchers also performed a subgroup analysis of the effect of atezolizumab on the molecular UBC subtype. ${ }^{14}$ Nivolumab showed the most effective drug response in the basal I subtype in the CheckMate 275 trial. $^{17}$ The luminal I subtype was low in PD-L1 and low in response to atezolizumab and nivolumab in tumour cells. ${ }^{1617}$

Genetic analysis of urothelial carcinoma found TCGA subtypes such as luminal and basal subtypes and TCGA clusters $\mathrm{I}-\mathrm{IV}^{10}{ }^{11}$ However, the classification of molecular subtyping presents various criteria for each study, making it difficult to standardise the classification of TCGA subtypes. ${ }^{10-1315}$ In the discussion of this classification, several researchers have defined BASQ as a molecular subtype classification of bladder cancer.

The basal type is associated with a high resistance to chemotherapy and poor prognosis. ${ }^{14}$ This molecular subtype of urothelial carcinoma is related to cell differentiation and is distinguished as basal and luminal type by a keratin marker. ${ }^{118}$ The basal type is characterised by a high-molecular-weight keratin, which represents the basal and stem cell compartment, and the luminal-type keratin represents the umbrella cell compartment. ${ }^{11} 18$ 
The recently presented neoadjuvant atezolizumab ABACUS $^{8}$ trial at American Society of Clinical Oncology 2018 was similar to ours, but the difference was that two cycles of neoadjuvant atezolizumab were performed ${ }^{89}$ In our study, three cycles of neoadjuvant atezolizumab are performed. According to a report of ABACUS ${ }^{8}$ trial in 2018, the PURE-01 study used three cycles of pembrolizumab and showed a pCR rate of $42 \%$. And the ABACUS study used two cycles of atezolizumab and showed a pCR rate of $31 \%$. We believe from the above two results and the characteristics of immunotherapy that there is an increase in effect as the cycle increases, and we expect three cycles to be more effective than two cycles. Seventy-five patients with T2-T4aN0M0 urothelial carcinoma were enrolled, of which $29 \%$ of the patients had pCR and $39 \%$ of them were downstaged with non-MIBC.$^{89}$ In PD-L1positive patient group, pCR was as high as $40 \% .^{89}$

There are a number of potential problems with this work: there is a lack of mature follow-up period that indicates survival outcome, and there is insufficient evidence for pT0 responses after immunotherapy to predict survival rates as in previous chemotherapy studies.

In conclusion, this study was designed to evaluate the effectiveness of the BASQ classification to select an effective target group for neoadjuvant atezolizumab treatment in patients with MIBC. This study will provide data that support a rational basis for selective neoadjuvant immunotherapy depending on the molecular characteristics of the individual tumour in the patient with bladder cancer, and it will be helpful in treating patients with MIBC by encouraging new neoadjuvant immunotherapy-related studies. We hope to reflect the results of personalised treatment according to the characteristics of individual tumours in patients with bladder cancer.

\section{TRIAL STATUS}

The protocol version number was ver.1.1. The first patient was enrolled on 13 August 2018, and a total of 18 patients have registered and are currently undergoing treatment (11 cases of basal type and 7 cases of luminal type). By August 2021, we expect that recruitment will be approximately completed.

\section{ETHICS AND DISSEMINATION}

The study protocol, information on Informed Consent Forms (online supplemental file 1) to be provided to the patient and relevant support information were reviewed and approved by the Institutional Review Board (IRB) of Seoul National University Hospital, Seoul, Republic of Korea (H 1806-051-950). Before the study began, patient recruitment data were approved by the IRB. Written consent to participate in the study was obtained prior to conducting the study-specific screening or evaluation. All screenings were completed and reviewed to ensure that patients met all eligibility criteria before treatment with neoadjuvant medication. Anonymous research results will be disseminated as a summary of research results between researchers, published manuscripts in peer-reviewed academic journals, presented abstracts, or presentations at conferences and academic meetings.

Contributors HDY, CWJ, CK, HK and JHK contributed to the concept and project planning. KCM contributed to administrative, technical or material support. JHK contributed to obtaining funding.

Funding The authors have not declared a specific grant for this research from any funding agency in the public, commercial or not-for-profit sectors.

Competing interests None declared.

Patient consent for publication Not required.

Provenance and peer review Not commissioned; externally peer reviewed.

Supplemental material This content has been supplied by the author(s). It has not been vetted by BMJ Publishing Group Limited (BMJ) and may not have been peer-reviewed. Any opinions or recommendations discussed are solely those of the author(s) and are not endorsed by BMJ. BMJ disclaims all liability and responsibility arising from any reliance placed on the content. Where the content includes any translated material, BMJ does not warrant the accuracy and reliability of the translations (including but not limited to local regulations, clinical guidelines, terminology, drug names and drug dosages), and is not responsible for any error and/or omissions arising from translation and adaptation or otherwise.

Open access This is an open access article distributed in accordance with the Creative Commons Attribution Non Commercial (CC BY-NC 4.0) license, which permits others to distribute, remix, adapt, build upon this work non-commercially, and license their derivative works on different terms, provided the original work is properly cited, appropriate credit is given, any changes made indicated, and the use is non-commercial. See: http://creativecommons.org/licenses/by-nc/4.0/.

ORCID iD

Ja Hyeon Ku http://orcid.org/0000-0002-0391-2342

\section{REFERENCES}

1 Donin NM, Lenis AT, Holden S, et al. Immunotherapy for the treatment of urothelial carcinoma. J Urol 2017;197:14-22.

2 Kates M, Sopko NA, Matsui H, et al. Immune checkpoint inhibitors: a new frontier in bladder cancer. World J Urol 2016;34:49-55.

$3 \mathrm{Kim}$ HS, Seo HK. Immune checkpoint inhibitors for urothelial carcinoma. Investig Clin Urol 2018;59:285-96.

4 Suzman DL, Agrawal S, Ning Y-M, et al. Fda approval summary: Atezolizumab or pembrolizumab for the treatment of patients with advanced urothelial carcinoma ineligible for cisplatin-containing chemotherapy. Oncologist 2019;24:563-9.

5 Gupta S, Gill D, Poole A, et al. Systemic immunotherapy for urothelial cancer: current trends and future directions. Cancers 2017;9. doi:10.3390/cancers9020015. [Epub ahead of print: 27 Jan 2017].

6 Massari F, Santoni M, di Nunno V, Nunno di V, et al. Adjuvant and neoadjuvant approaches for urothelial cancer: updated indications and controversies. Cancer Treat Rev 2018;68:80-5.

7 Necchi A, Anichini A, Raggi D, et al. Pembrolizumab as neoadjuvant therapy before radical cystectomy in patients with muscle-invasive urothelial bladder carcinoma (PURE-01): an open-label, single-arm, phase II study. J Clin Oncol 2018;36:3353-60.

8 Powles T, Kockx M, Rodriguez-Vida A, et al. Clinical efficacy and biomarker analysis of neoadjuvant atezolizumab in operable urothelial carcinoma in the ABACUS trial. Nat Med 2019;25:1706-14.

9 Pichler R, Horninger W, Heidegger I. ASCO 2018: highlights of urothelial cancer and prostate cancer. Memo 2018;11:284-90.

10 Cancer Genome Atlas Research Network. Comprehensive molecular characterization of urothelial bladder carcinoma. Nature 2014;507:315-22.

11 Choi W, Porten S, Kim S, et al. Identification of distinct basal and luminal subtypes of muscle-invasive bladder cancer with different sensitivities to frontline chemotherapy. Cancer Cell 2014;25:152-65.

12 Hoadley KA, Yau C, Wolf DM, et al. Multiplatform analysis of 12 cancer types reveals molecular classification within and across tissues of origin. Cell 2014;158:929-44.

13 Lindgren D, Frigyesi A, Gudjonsson S, et al. Combined gene expression and genomic profiling define two intrinsic molecular subtypes of urothelial carcinoma and gene signatures for molecular grading and outcome. Cancer Res 2010;70:3463-72. 
14 Lerner SP, McConkey DJ, Hoadley KA, et al. Bladder cancer molecular taxonomy: summary from a consensus meeting. Bladder Cancer 2016;2:37-47.

15 Volkmer J-P, Sahoo D, Chin RK, et al. Three differentiation states risk-stratify bladder cancer into distinct subtypes. Proc Natl Acad Sci U S A 2012;109:2078-83.

16 Rosenberg JE, Hoffman-Censits J, Powles T, et al. Atezolizumab in patients with locally advanced and metastatic urothelial carcinoma who have progressed following treatment with platinum-based chemotherapy: a single-arm, multicentre, phase 2 trial. Lancet 2016;387:1909-20.

17 Sharma P, Retz M, Siefker-Radtke A, et al. Nivolumab in metastatic urothelial carcinoma after platinum therapy (CheckMate 275): a multicentre, single-arm, phase 2 trial. Lancet Oncol 2017;18:312-22.

18 Perou CM, Sørlie T, Eisen MB, et al. Molecular portraits of human breast tumours. Nature 2000;406:747-52. 